\title{
Management of jugular bulb injury during drilling of the internal auditory canal (ICA) for vestibular schwannoma surgery
}

\author{
Sajjad Muhammad ${ }^{\mathrm{a}, *}$, Martin Lehecka $^{\mathrm{a}}$, Saku T. Sinkkonen ${ }^{\mathrm{b}}$, Mika Niemelä \\ ${ }^{a}$ Department of Neurosurgery, University of Helsinki and Helsinki University Hospital, Helsinki, Finland \\ ${ }^{\mathrm{b}}$ Department of Otolaryngology, University of Helsinki and Helsinki University Hospital, Helsinki, Finland
}

\section{A R T I C L E I N F O}

\section{Keywords:}

Jugular bulb injury

Vestibular schwannoma

Surgery

Technical aspects

\begin{abstract}
A B S T R A C T
The retrosigmoid approach for vestibular schwannoma surgery has remained the standard approach by most neurosurgeons. Drilling the posterior wall of the internal auditory meatus (IAM) is an essential step in removing the intrameatal tumor. During IAM drilling, three anatomical structures can be encountered, including the posterior semicircular canal, vestibular aqueduct, and jugular bulb. Any of these can be injured during drilling, especially if the jugular bulb lies above the inferior edge of the IAM. Although IAM drilling is performed in most vestibular schwannoma surgeries, information on how to manage complications such as jugular bulb injury is lacking. Here we use an intraoperative video to demonstrate how to manage the inadvertent injury to the jugular bulb in order to avoid massive blood loss. We present a case of a 39-year-old woman with hearing loss, diagnosed with a cerebellopontine angle mass extending into the IAM. Surgery was required due to tumor progression. We used the retrosigmoid approach to access the tumor. During IAM drilling, the jugular bulb was injured. A thin layer of bone wax was applied under continuous suction. The margins of the wax were then gently compressed with a dissector; great care was taken to avoid pushing the wax into the jugular bulb. Excess bone wax was removed (video 1). A small diamond drill $(2 \mathrm{~mm})$ was used for further drilling. Our instructional video shows the surgical approach, microsurgical anatomy, and technical aspects of managing massive bleeding from jugular bulb injury. It should therefore be helpful for young neurosurgeons.
\end{abstract}

\section{Conflict of interest}

None.

\section{Appendix A. Supplementary data}

Supplementary data to this article can be found online at https:// doi.org/10.1016/j.amjoto.2019.01.004.

\footnotetext{
Abbreviations: IAM, Internal auditory meatus; IAC, Internal auditory canal

* Corresponding author at: University of Helsinki and Helsinki University Hospital, Helsinki, Finland.

E-mail address: ext-sajjad.muhammad@hus.fi (S. Muhammad).
} 